\title{
On the electrophoretic mobility of succinoglycan modelled as a spherical polyelectrolyte: From Hermans-Fujita theory to charge regulation in multi-component electrolytes
}

\author{
Reghan J. Hill ${ }^{\mathrm{a}, 1, *}$ \\ ${ }^{a}$ Department of Chemical Engineering, McGill University, \\ Montreal, Quebec, H3A OC5, Canada
}

\begin{abstract}
Literature interpretations of the electrophoretic mobility of spherical polyelectrolytes are revisited using the capillary-electrophoresis data of Duval et al. (Biomacromolecules, 7, 2818-2826, 2006) for the extracellular polysaccharide succinoglycan as an example. Subtle changes in the polyelectrolyte mobility have recently been attributed to new electrokinetic theories that feature multi-component electrolytes, charge regulation, and the so-called polarization and relaxation phenomena. However, these calculations exhibit several unusual trends that have yet to be explained, and so the conclusions drawn from them are controversial. Here, independent computations strengthen conclusions drawn from the original model of Duval et al., i.e., the discrepancies between experiments and all the presently available electrokinetic theories reflect changes in the conformation of succinoglycan arising from changes in the electrolyte $\mathrm{pH}$ and ionic strength.
\end{abstract}

Keywords:

\section{Introduction}

This study addresses subtle features of the electrophoretic mobility versus ionic-strength relationship for succinoglycan macromolecules. Duval et al. [2]

\footnotetext{
${ }^{*}$ Corresponding author

Email address: reghan.hill@mcgill.ca (Reghan J. Hill)

${ }^{1}$ Tel. 5143986897
} 
measured this relationship and interpreted the data using electrokinetic models for spherical and rod-like polyelectrolytes, explicitly addressing changes in the charge arising from changes in the $\mathrm{pH}$ and ionic strength of the electrolyte, hereafter termed charge regulation. Their model furnished estimates of the protolytic binding site density and equilibrium dissociation constants. Moreover, the theory advanced the well-known Hermans-Fujita model to account for non-linear electrostatics (via the non-linear PoissonBoltzmann equation), ion-concentration perturbations - also termed doublelayer-polarization (DLP) - and charge regulation.

However, two recent theoretical studies by Yeh and coworkers $[11,10]$ (both using finite-element (FE) modelling software to compare models with an without DLP) argued that new electrokinetic models elucidate the subtle changes in the mobility-ionic-strength relationship (elaborated upon below) not captured by the model of Duval et al. [2] Both Yeh and coworkers' theoretical interpretations predict that DLP increases the magnitude of the mobility at high ionic strengths, as evidenced by a crossing of the mobility versus ionic strength relationships undertaken with and without DLP. As discussed in the SI, this unusual behaviour is contrary to the general expectation that DLP decreases the mobility magnitude. Moreover, the charge-regulation model of Yeh et al. [11] is not fundamentally different from the model of Duval et al. [2], since both include charge regulation closures, and both account for DLP (the latter attribute seems to have been overlooked by Yeh and coworkers).

Attributing the qualitative differences between the theoretical calculations to new physics is controversial, and surely demands further investigation. The anomalies in question might be considered minor and, therefore, inconsequential from a practical perspective. However, such nuances have been used to highlight new physical insights and to motivate intricate mathematical models for interpreting experiments, e.g., as tools for parameter fitting. Knowledge of whether differences between model predictions can be attributed to new physics or, perhaps, computational artifacts is clearly important.

The challenges of accurately solving particle-electrophoresis models are well known [8]. Indeed, these motivate analytical approximate theories, such as the Hermans-Fujita formula, and specialized computational methods, as implemented below. The solution methodology adopted in this study will be demonstrated to agree with the calculations of Duval et al. [2] (figure 1) and the Hermans-Fujita theory (figure 2). While the former tests general aspects of the electrokinetic model, including charge regulation, non-linear 
electrostatics, and DLP, the latter tests computational fidelity under the most challenging conditions where disparities in particle and diffuse-layer length scales are very large $(\kappa a \gg 1)$.

\section{Electrokinetic model}

The calculations reported here were undertaken using the same methodology that underlies the MPEK package, which emerged from early attempts to capture the electrostatic non-linearities and polarization and relaxation dynamics for soft colloidal spheres with an impenetrable core and a soft, possibly charged, permeable corona $[7,6]$. Here, it was modified to include proton-donating and proton-accepting sites. Accordingly, for the zwitterionic regulation model of Yeh and coworkers, the immobile charge density $\rho_{f}$ arising from the groups $\mathrm{X}_{1} \mathrm{H}$ (acid, $-\mathrm{COOH}$ ) and $\mathrm{X}_{2}$ (base, $-\mathrm{NH}_{2}$ ) associated with $\mathrm{H}^{+}$was prescribed according to equilibria

$$
\mathrm{X}_{1} \mathrm{H} \rightleftharpoons \mathrm{X}_{1}^{-}+\mathrm{H}^{+} \text {and } \mathrm{X}_{2} \mathrm{H}^{+} \rightleftharpoons \mathrm{X}_{2}+\mathrm{H}^{+}
$$

with equilibrium constants $K_{1}=\left[\mathrm{X}_{1}^{-}\right]\left[\mathrm{H}^{+}\right] /\left[\mathrm{X}_{1} \mathrm{H}\right]$ and $K_{2}=\left[\mathrm{X}_{2}\right]\left[\mathrm{H}^{+}\right] /\left[\mathrm{X}_{2} \mathrm{H}^{+}\right]$, and binding-site densities $n_{f, 1}^{*}=\left[\mathrm{X}_{1} \mathrm{H}\right]+\left[\mathrm{X}_{1}^{-}\right]$and $n_{f, 2}^{*}=\left[\mathrm{X}_{2} \mathrm{H}^{+}\right]+\left[\mathrm{X}_{2}\right]$. It follows that

$$
\left[\mathrm{X}_{1}^{-}\right]=\frac{n_{f, 1}^{*}}{1+\left[\mathrm{H}^{+}\right] / K_{1}} \text { and }\left[\mathrm{X}_{2} \mathrm{H}^{+}\right]=\frac{n_{f, 2}^{*}}{1+K_{2} /\left[\mathrm{H}^{+}\right]}
$$

so the immobile charge density for this charge-regulation model can be written

$$
\rho_{f}=-\frac{e n_{f, 1}^{*}}{1+\left[\mathrm{H}^{+}\right] / K_{1}}+\frac{e n_{f, 2}^{*}}{1+K_{2} /\left[\mathrm{H}^{+}\right]} .
$$

Details of the electrokinetic model, which addresses a much more general class of charge-regulating spherical nanoparticulates comprising a rigid, impenetrable core and a porous corona, are available elsewhere [4]. To model a porous sphere, the radius of the nanoparticle core $a_{c}$ must be set to a value that is much smaller than the nominal thickness $L$ of the corona. In this limit, the charge and hydrodynamic drag of the vanishingly small core become negligible to the charge and drag on the corona. In this study, a porous sphere is prescribed using a Stokes-segment density profile

$$
n_{s}(r)=n_{s, 0} 0.5 \operatorname{erfc}\left[-\left(r-L-a_{c}\right) / \delta\right]
$$


Table 1: Independent model parameters (others are provided in the figure captions). ${ }^{\dagger}$ The mobility and drag coefficient are practically independent of these parameters because $a_{c} \ll L$, so the core-shell particle mimics a spherical polyelectrolyte.

\begin{tabular}{|c|c|c|c|}
\hline parameter & symbol & value & units \\
\hline core surface-charge density $^{\dagger}$ & $\sigma_{c}$ & 0 & $\mu \mathrm{C} \mathrm{cm}^{-2}$ \\
\hline core radius $^{\dagger}$ & $a_{c}$ & 0.108 & $\mathrm{~nm}$ \\
\hline temperature & $T$ & 298 & $\mathrm{~K}$ \\
\hline core relative permittivity ${ }^{\dagger}$ & $\epsilon_{c}$ & $10^{-8}$ & - \\
\hline solvent relative permittivity & $\epsilon_{s}$ & 78.5 & - \\
\hline solvent viscosity & $\eta$ & $8.9 \times 10^{-4}$ & Pa s \\
\hline Stokes segment profile & $n_{s}(r)$ & Eqn. (1) & - \\
\hline corona charge profile & $n_{f, 1}^{*}(r)$ & Eqn. (2) & - \\
\hline corona charge profile & $n_{f, 2}^{*}(r)$ & Eqn. (3) & - \\
\hline Stokes segment parameter & $n_{s, 0}$ & 19.8 & M \\
\hline Stokes segment parameter & $L / a_{c}$ & 99 & - \\
\hline Stokes segment parameter & $\delta / a_{c}$ & 0.99 & - \\
\hline corona charge parameter & $L / a_{c}$ & 99 & - \\
\hline corona charge parameter & $\delta / a_{c}$ & 0.99 & - \\
\hline
\end{tabular}

and accompanying radial binding-site profiles

$$
\begin{aligned}
& n_{f, 1}^{*}(r)=n_{f, 1,0}^{*} 0.5 \operatorname{erfc}\left[-\left(r-L-a_{c}\right) / \delta\right] \\
& n_{f, 2}^{*}(r)=n_{f, 2,0}^{*} 0.5 \operatorname{erfc}\left[-\left(r-L-a_{c}\right) / \delta\right] .
\end{aligned}
$$

With $\delta \ll L$, this approximates very well the perfectly step-like permeability and charge distribution adopted in the Hermas-Fujita theory and the calculations of Duval et al. [2], Yeh et al. [10] and Yeh et al. [11] As summarized in table 1 , the calculations were undertaken with $\delta=0.01 L$ and $L=99 a_{c}$ (so the nominal spherical polyelectrolyte radius $a=a_{c}+L=100 a_{c} \approx 1.01 L$ ). The nominal Stokes-resistance center and immobile charge-concentration parameters, $n_{s, 0}, n_{f, 1,0}^{*}$ and $n_{f, 2,0}^{*}$, were varied to expedite direct comparisons with Yeh and coworkers, prescribing $n_{s, 0}$ and $a_{s}$ so that $\ell^{-2}=6 \pi a_{s} n_{s, 0}$.

Because the core is sufficiently small and weakly charged, the forces on the (spherical) polyelectrolyte are practically independent of the core (size, dielectric constant, and surface charge). This is verified below by comparison to independent numerical calculations and analytical theory. Note, however, 
Table 2: Electrolyte-ion concentrations $\left(n_{j}^{\infty}\right)$ and the limiting ion conductivities $\left(\lambda_{j}\right)$ used to prescribe ion mobilities.

\begin{tabular}{lll}
\hline ion & $n_{j}^{\infty}(\mathrm{M})$ & $\lambda_{j}\left(\mathrm{~S} \mathrm{~cm}^{2} \mathrm{~mol}^{-1}\right)$ \\
\hline $\mathrm{H}^{+}$ & $n_{1}^{\infty}=10^{-} \mathrm{pH}$ & 350 \\
\hline $\mathrm{Cl}^{-}$ & $n_{2}^{\infty}(\mathrm{pH}>7)$ & 76.4 \\
$\mathrm{Na}^{+}$ & $n_{3}^{\infty}=-n_{1}^{\infty}+n_{2}^{\infty}+n_{4}^{\infty}(\mathrm{pH}>7)$ & 50.1 \\
\hline $\mathrm{Cl}^{-}$ & $n_{2}^{\infty}=n_{1}^{\infty}+n_{3}^{\infty}-n_{4}^{\infty}(\mathrm{pH}<7)$ & 76.4 \\
$\mathrm{Na}^{+}$ & $n_{3}^{\infty}(\mathrm{pH}<7)$ & 50.1 \\
\hline $\mathrm{OH}^{-}$ & $n_{4}^{\infty}=10^{-14+\mathrm{pH}}$ & 199 \\
\hline
\end{tabular}

that a vanishing of the core's influence on the polyelectrolyte mobility when $a_{c} \ll a$ is readily anticipated from the following physical and scaling considerations.

A sufficiently small core diminishes its hydrodynamic size and charge to values that are comparable to or smaller than of the (many more) segments that comprise the corona. Since the hydrodynamic drag force on the core translating at velocity $V$ is $O\left(-\eta a_{c} V\right)$, and the electrical force is $O\left(\sigma a_{c}^{2} E\right)$, these are obviously negligible when compared to the $O(-\eta a V)$ and $O\left(\rho_{f} a^{3} E\right)$ hydrodynamic and electrical forces on the corona when $a_{c} \ll a$ and $\kappa a \ll 1$. When $\kappa a \gg 1$, the electric field drives an $O\left(-\rho_{f} \ell^{2} E / \eta\right)$ electroosmotic flow velocity $U_{e o}$ through the polyelectrolyte; however, the hydrodynamic drag on the segments precisely balances the electrical force, and so the net electricfield-induced force on the polyelectrolyte is dominated by the external viscous flow, which furnishes an $O\left(-\eta a U_{e o}=a \rho_{f} \ell^{2} E\right)$ force, and, thus, an $O\left(\rho_{f} \ell^{2} / \eta\right)$ electrophoretic mobility $V / E$ when $\kappa a \gg 1$, as predicted by the HermansFujita theory in this limit.

The forces from the MPEK methodology are customarily computed from the far-field velocity disturbances in an unbounded electrolyte [8, 7]. To avoid unnecessary controversy over the force-evaluation method, the forces were also calculated from direct integrations of the electrical and hydrodynamic forces on the corona/polyelectrolyte, as detailed by Hill [4]. Both methods furnished the same mobilities (to numerical discretization accuracy), and also reproduced the Hermans-Fujita theory for uniform, weakly charged spheres (fixed charge), without DLP. Studies of DLP in multi-component electrolytes with charge regulating coronas and gels (for nanoparticle gel electrophore- 
sis) have recently been undertaken by Hill [4] and Hill [5]. These provide direct comparisons of computations undertaken with and without DLP, also assessing the influence of electrolyte-ion mobilities on the particle drag coefficient (elucidating electroviscous effects resulting from polarization), as well quantifying how numerical errors scale with the computational extent, grid resolution, and force-evaluation method.

\section{Results and discussion}

The MPEK package furnishes many useful diagnostics, e.g., drag, coefficient, polarizability, and conductivity increment. Here we examine the dimensionless electrophoretic mobility

$$
M^{*}=\frac{V}{E} \frac{\eta e}{\epsilon_{s} \epsilon_{0} k_{B} T},
$$

where $V$ is the (signed) electrophoretic particle velocity in response to an electric field with strength $E$. Note that the customary factor of $3 / 2$ has been discarded to expedite a direct comparison with Yeh and coworkers. The methodology was first tested by computing spherical polyelectrolyte mobilities using the charge-regulation model of Duval et al. [2] As shown in figure 1, these calculations (which account for charge regulation, non-linear electrostatics, and DLP) are in excellent agreement over the full range of ionic strengths reported by Duval et al. [2].

Additional tests were undertaken by direct comparison with the wellknown Hermans-Fujita theory (see also Ohshima [9]) over an unprecedented range of fixed charge densities and ionic strengths. The Hermans-Fujita formula can be written

$$
\frac{V}{E} \approx \frac{\rho_{f} \ell^{2}}{\eta}\left[1+\left(\frac{a}{\ell}\right)^{2} \frac{1+e^{-2 \kappa a}}{9+3(\kappa a)^{2}}\right],
$$

where $\rho_{f}$ is the fixed/immobile charge density, and $\ell$ and $\kappa^{-1}$ are, respectively, the Brinkman and Debye lengths. The dimensional pre-factor is the limiting mobility at high ionic strength when $\kappa a \gg 1$. As expected, the results presented in figure 2 are in excellent agreement when the electrostatic potential is low enough to justify the Debye-Hückel approximation and neglect of DLP. Note that the (sometimes significant) differences arising from non-linear electrostatics and DLP are consistent with expectations 


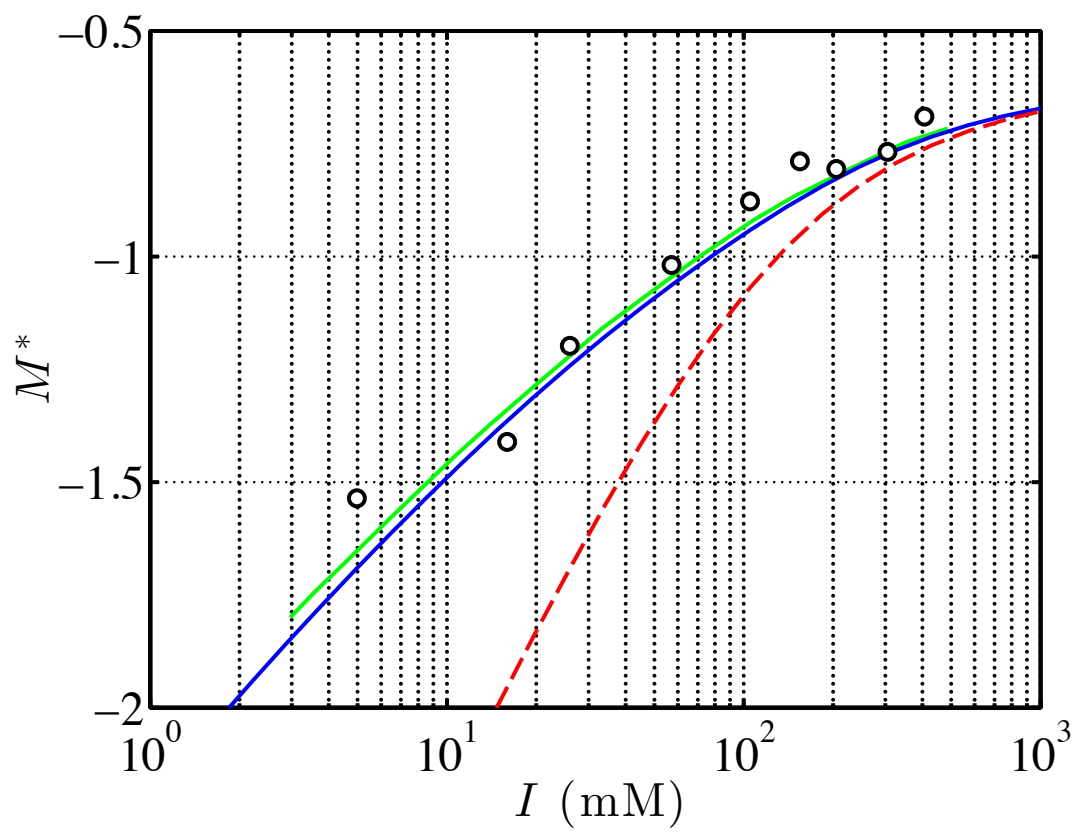

Figure 1: Electrophoretic mobility compared with Duval et al. [2] (charge regulation, nonlinear electrostatics, DLP): $a=10.8 \mathrm{~nm}, \ell=0.7 \mathrm{~nm}, n_{f, 1,0}^{*}=0.239 \mathrm{M}, \mathrm{pK}_{1}=4.58$ and $\mathrm{pK}_{2}=8.60$ at $\mathrm{pH}=10.3$. The green line is the data labelled as 'rigorous theory' from figure $3 \mathrm{~B}$ of Duval et al. [2], which they evaluated using the computational methodology of Duval and Ohshima [1]; the blue solid (red dashed) lines are calculations using the same charge-regulating electrokinetic model of Duval et al. [2] (two acid-dissociation moieties) evaluated with (without) DLP. Symbols are the experimental data of Duval et al. [2] 
gleaned from soft core-shell nanoparticles [7] and the foregoing comparison with Duval et al. [2] Moreover, the differences between the numerically exact calculations without DLP (red dashed lines) and Eqn. (4) (black dash-dotted lines) are because the Debye-Hückel approximation (in the analytical approximation) breaks down as the magnitude of the electrostatic potential inside the polyelectrolyte increases with decreasing ionic strength (decreasing $\kappa a$ ).

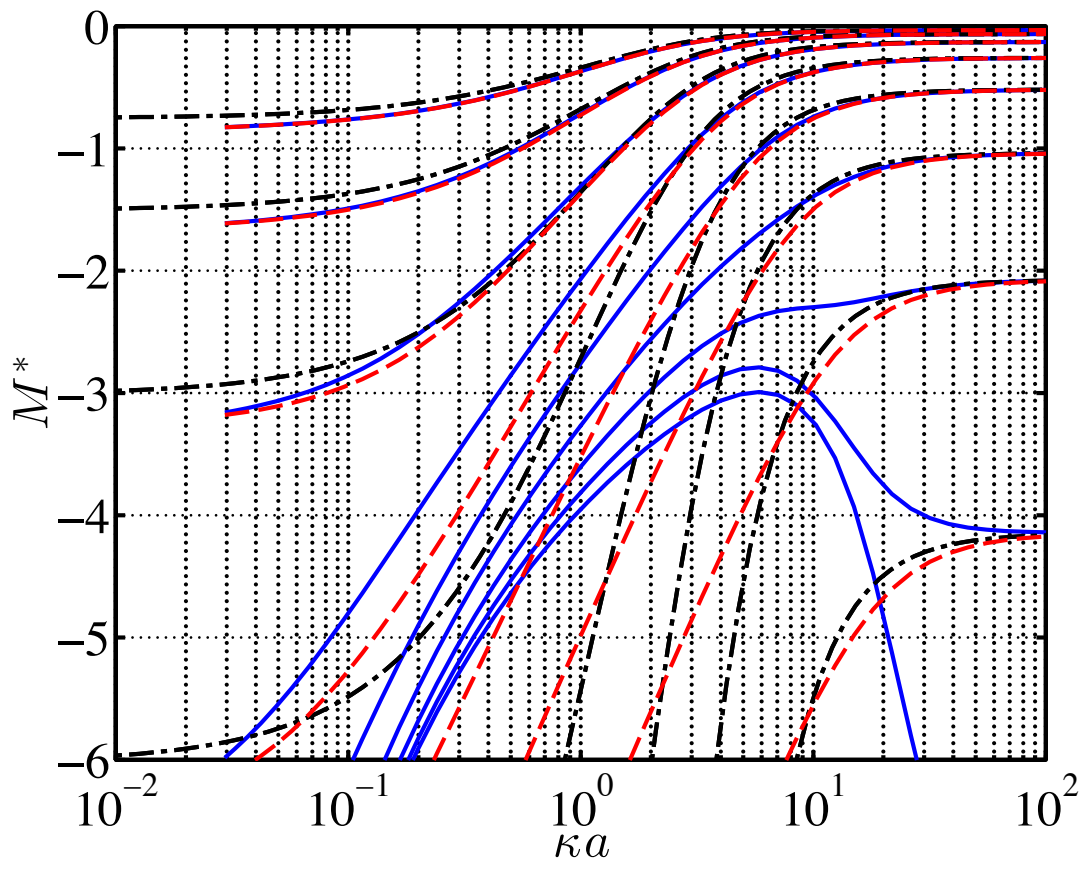

Figure 2: Spherical polyelectrolyte electrophoretic mobility versus scaled reciprocal Debye length $\kappa a: a=20 \mathrm{~nm}, \ell=2 \mathrm{~nm}$, and fixed charge densities $\rho_{f}=1,2,4, \ldots 128,256 \mathrm{mM}$ (top to bottom). The blue solid (dashed red) lines are calculations $(\delta=L / 100$ with electrolytes containing $\mathrm{H}^{+}, \mathrm{Cl}^{-}, \mathrm{Na}^{+}$and $\mathrm{OH}^{-}$ions at $\mathrm{pH}=7$ ) with (without) DLP. The black dash-dotted lines are Eqn. (4) (Hermans-Fujita theory: Debye-Hückel approximation without DLP).

Finally, we turn to the electrophoretic mobilities of Yeh et al. [11] General model parameters are summarized in tables 1 and 2 with other parameters (specific to particular calculations) provided in figure captions. To expedite comparisons with Yeh et al. [11], all the calculations were undertaken with four electrolyte ion species. The concentrations of $\mathrm{H}^{+}$and $\mathrm{OH}^{-}$were set according to the prescribed $\mathrm{pH}$, with the concentration of $\mathrm{Na}^{+}\left(\mathrm{Cl}^{-}\right)$chosen 
to vary the bulk ionic strength $I$ when $\mathrm{pH}<7(>7)$, and the concentration of $\mathrm{Cl}^{-}\left(\mathrm{Na}^{+}\right)$calculated to ensure an electro-neutral bulk electrolyte when $\mathrm{pH}<7(>7)$. Note that there is a lower limit to the ionic strength at each $\mathrm{pH}$, which increases as the $\mathrm{pH}$ deviates from 7 .

The mobility versus ionic-strength relationship is shown in figure 3 using the same parameters as Yeh et al. [11] The results in figure 2 of Yeh et al. [11] (available in the SI) were calculated with $\mathrm{pH}=10.3$ (as also in the experiments to which they compared their calculations). It is therefore important to note that the pair of curves in figure 3 that begin at an ionic strength $0.2 \mathrm{mM}$ are the ones to be compared to Yeh et al. [11] Note that the ionic strength in figure 3 has been extended to $1 \mathrm{M}$ to avoid unnecessary controversy over the way in which the mobility approaches the high-ionic-strength limit.

The calculations with and without DLP plateau at high ionic strength to the value furnished by Eqn. (4) in this limit (horizontal dash-dotted line). Consistent with the general expectation stated in the introduction (and discussed in the SI), there is no crossing of the curves computed with and without DLP. Thus, contrary to the predictions of Yeh et al. [11] and Yeh et al. [10], the magnitude of the mobility is indeed diminished by DLP at high ionic strength, and this attenuation is amplified by the increasing electrostatic potential that accompanies a decrease in the electrolyte ionic strength.

Note that $\mathrm{pH}=10.3$ is considerably higher than the values of $\mathrm{pK}_{1}$ and $\mathrm{pK}_{2}$ prescribed for $\mathrm{H}^{+}$(see table 2), so under these conditions the charge regulation is weak. Nevertheless, the additional calculations presented in figure 3 with four lower $\mathrm{pH}$ values confirm that the charge decreases with decreasing pH. Because the electrostatic potential decreases with the decreasing charge that accompanies the change in $\mathrm{pH}$, this attenuates the role of DLP. At $\mathrm{pH}=4$ and 5, for example, the difference between the solid (with DLP) and dashed (without DLP) curves is practically null. Again, all the curves asymptote to the limiting mobilities of Eqn. (4), as demonstrated in figure 2 for polyelectrolytes bearing a fixed charge.

\section{Conclusions}

The calculations undertaken here show that the unusual 'bumps' and crossings of the electrophoretic mobility versus ionic-strength relationship predicted by Yeh et al. [11] and Yeh et al. [10] cannot be attributed to the electrokinetic models. The present calculations validate those of Duval 


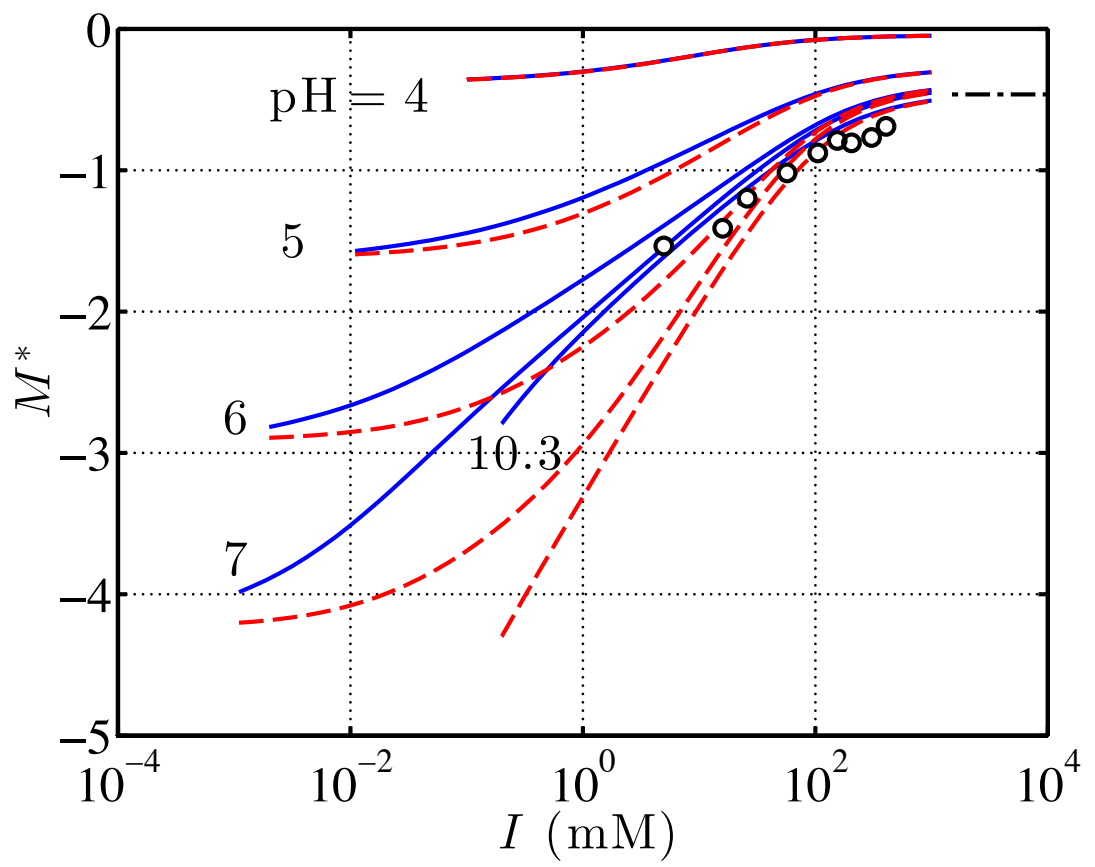

Figure 3: Electrophoretic mobility for succinoglycan versus ionic strength for an electrolyte comprising $\mathrm{H}^{+}, \mathrm{Cl}^{-}, \mathrm{Na}^{+}$and $\mathrm{OH}^{-}$and other parameters as prescribed by Yeh et al. [11]: $a=10.8 \mathrm{~nm}, \ell=0.635 \mathrm{~nm}, n_{f, 1,0}^{*}=0.213 \mathrm{M}, n_{f, 2,0}^{*}=0.026 \mathrm{M}, \mathrm{pK}_{1}=4.58$ and $\mathrm{pK}_{2}=8.60$. Other parameters are listed in tables 1 and 2. Solid (dashed) lines are calculations with (without) DLP. The dash-dotted line is the value furnished by Eqn. (4) at high ionic strength for the fully dissociated polyelectrolyte $\left(M^{*} \approx-0.46\right)$, and the symbols are experimental data from Duval et al. [2] at $\mathrm{pH}=10.3$. 
et al. [2], the analytical theory of Hermans [3], and indeed other numerical calculations undertaken by Yeh and coworkers, albeit in other regions of the parameter space (not compared to the data and calculations of Duval et al.). Thus, the anomalous electrokinetic interpretations must be attributed to computational artifacts in the experimental region of the parameter space or, perhaps, oversights in the fitting and reporting of the model parameters. The present calculation methodology (i) has 2nd- or 3rd-order convergence (depending on the force-evaluation methodology) when increasing the radial extent of the computational domain [4], (ii) adopts a highly adaptive grid, and (iii) captures the far-field asymptotic decay of the decaying perturbation fields (ion concentration, electrostatic potential, and fluid velocity). Thus, its high accuracy for a broad class of spherical core-shell nanoparticles provides a useful benchmark with which to test new models and solution methodologies.

\section{Acknowledgements}

Financial support from an NSERC Discovery Grant is greatfully acknowledged.

\section{References}

[1] Duval, J.F.L., Ohshima, H., 2006. Electrophoresis of Diffuse Soft Particles. Langmuir 22, 3533-3546.

[2] Duval, J.F.L., Slaveykova, V.I., Hosse, M., Buffle, J., Wilkinson, K.J., 2006. Electrohydrodynamic Properties of Succinoglycan as Probed by Fluorescence Correlation Spectroscopy, Potentiometric Titration and Capillary Electrophoresis. Biomacromolecules 7, 2818-2826.

[3] Hermans, J.J., 1955. Sedimentation and electrophoresis of porous spheres. J. Polym. Sci. 18, 527-534.

[4] Hill, R.J., 2015a. Corona charge regulation in nanoparticle electrophoresis. Proc. R. Soc. A 471, 20150522.

[5] Hill, R.J., 2015b. Hydrogel charge regulation and electrolyte ionconcentration perturbations in nanoparticle gel-electrophoresis. Proc. R. Soc. A 471, 20150523. 
[6] Hill, R.J., Saville, D.A., 2005. 'Exact' solutions of the full electrokinetic model for soft spherical colloids: Electrophoretic mobility. Colloids Surfaces A 267, 31-49. doi:10.1016/j.colsurfa.2005.06.035.

[7] Hill, R.J., Saville, D.A., Russel, W.B., 2003. Electrophoresis of spherical polyer-coated colloidal particles. J. Colloid Interface Sci. 258, 56-74.

[8] O'Brien, R.W., White, L.R., 1978. Electrophoretic mobility of a spherical colloidal particle. J. Chem. Soc. Faraday Trans. 74, 1607-1626.

[9] Ohshima, H., 1994. Electrophoretic mobility of soft particles. J. Colloid Interf. Sci. 163, 474-483.

[10] Yeh, L.H., Liu, K.L., Hsu, J.P., 2012a. Importance of Ionic Polarization Effect on the Electrophoretic Behavior of Polyelectrolyte Nanoparticles in Aqueous Electrolyte Solutions. J. Phys. Chem. C 116, 367-373.

[11] Yeh, L.H., Tai, Y.H., Wang, N., Hsu, J.P., Qian, S., 2012b. Electrokinetics of $\mathrm{pH}$-regulated zwitterionic polyelectrolyte nanoparticles. Nanoscale 4, 7575-7584. 